\title{
New formulae for magnetic relative helicity and field line helicity
}

\author{
Jean-Jacques Aly \\ AIM, Unité Mixte de Recherche CEA - CNRS - Université Paris VII, UMR n ${ }^{\circ} 7158$, \\ Centre d'Etudes de Saclay, F-91191 Gif sur Yvette Cedex, France \\ E-mail: jean-jacques.aly@cea.fr
}

\begin{abstract}
We consider a magnetic field $\mathbf{B}$ occupying the simply connected domain $D$ and having all its field lines tied to the boundary $S$ of $D$. We assume here that $\mathbf{B}$ has a simple topology, i.e., the mapping $\mathbf{M}$ from positive to negative polarity areas of $S$ associating to each other the two footpoints of any magnetic line, is continuous. We first present new formulae for the helicity $H$ of $\mathbf{B}$ relative to a reference field $\mathbf{B}_{r}$ having the same normal component $B_{n}$ on $S$, and for its field line helicity $h$ relative to a reference vector potential $\mathbf{C}_{r}$ of $\mathbf{B}_{r}$. These formulae make immediately apparent the well known invariance of these quantities under all the ideal MHD deformations that preserve the positions of the footpoints on $S$. They express indeed $h$ and $H$ either in terms of $\mathbf{M}$ and $B_{n}$, or in terms of the values on $S$ of a pair of Euler potentials of B. We next show that, for a specific choice of $\mathbf{C}_{r}$, the field line helicity $h$ of $\mathbf{B}$ fully characterizes the magnetic mapping $\mathbf{M}$ and then the topology of the lines. Finally, we give a formula that describes the rate of change of $h$ in a situation where the plasma moves on the perfectly conducting boundary $S$ without changing $B_{n}$ and/or non-ideal processes, described by an unspecified term $\mathbf{N}$ in Ohm's law, are at work in some parts of $D$.
\end{abstract}

Keywords: MHD, Magnetic helicity, Solar and stellar magnetic fields 


\section{Introduction}

The helicity of a magnetic field B occupying some domain $D$ of space is a global physical quantity whose importance in magnetohydrodynamics (MHD) stems from two essential properties (Woltjer 1958, Moffatt 1969): it is conserved when the field suffers an arbitrary ideal MHD deformation, thus constrained by the frozen-in law, and it gives information about the topology of the magnetic lines of $\mathbf{B}$. However, it is only defined when $\mathbf{B}$ has a vanishing normal component $B_{n}$ on the boundary $S$ of $D$. If this condition is not satisfied, as happens to be the case in many situations one has to deal with in astrophysics, it is still possible to construct a meaningful notion of helicity, but to do so requires introducing an external ingredient: a reference field $\mathbf{B}_{r}$ that has the same flux distribution as $\mathbf{B}$ on $S\left(\mathbf{B}_{r}\right.$ being most generally chosen to be the unique potential field satisfying that boundary condition). The new helicity, $H$, is defined relatively to that reference field and it has the same interesting properties as the original one (Berger and Field 1984): it is also an ideal MHD invariant, being conserved when the field is deformed under the frozen-in constraint while keeping its footpoints on $S$ at their initial positions, and it has also a topological meaning. By now, $H$ has become a basic tool in the study of astrophysical plasmas. In solar physics, for instance, it is used to deduce from observations important information on the nature of the mechanisms driving the spectacular coronal mass ejections originating from the corona and on the nature of the dynamo mechanism at work inside the Sun (for recent reviews, see, e.g., Démoulin and Pariat 2009, Pevtsov et al 2014).

Field line helicity relative to a vector potential of reference, $h$, is a function defined on the set of individual field lines of a field $\mathbf{B}$. As such, it provides a much more detailed description of the properties of $\mathbf{B}$ than the helicity $H$, which can be recovered from it by performing a flux-weighted integration. $h$ was first introduced by Berger (1988), who proved in particular its invariance under ideal MHD deformations preserving the positions of the footpoints on $S$. Later on, however, it seems to have been forgotten for a while, before knowing a revival with the most recent work of Yeates and Hornig (2014, and references therein), who renamed it "topological flux function" and extended our knowledge of its physical properties. Since then, evidences have accumulated that $h$ should be a precious tool for studying magnetic reconnection (Russell et al 2015) and the evolution of the solar magnetic field (Yeates and Hornig 2016). Hence it appears worth continuing to explore the properties of that quantity.

Some general issues that seem to be currently of interest for the development of

the two concepts introduced above and their practical applications to astrophysical problems, are the following ones:

(i) The topological invariance of the helicity $H$ and of the line helicity $h$ does not appear obvious if we just take a simple look at the standard expressions that are used to define them. Is it possible to derive alternative formulae exhibiting this invariance in a fully explicit way? Such formulae, valid in some specific geometrical settings, have already been proposed (Berger 1986, Aly 2014, Prior and Yeates 
2014) and they proved to be useful to get a better understanding of the physical meaning of $H$ and $h$.

(ii) By the invariance property recalled above, two fields that can be ideally deformed into each other without moving their footpoints - they are said to be topologically equivalent - have the same field line helicity. Does the converse property hold true, i.e., can we deduce from the fact that two fields have the same field line helicity that they are topologically equivalent? This problem was first addressed and solved for a specific type of magnetic field by Yeates and Hornig (2014, and references therein).

(iii) In the case where the magnetic field $\mathbf{B}$ evolves in time, with its footpoints being dragged along on the boundary by plasma motions and/or with non-ideal processes acting in the bulk of $D$, what is the rate of change of the helicity $h$ of a given line? This point is certainly important when considering the relaxation (involving turbulent magnetic reconnection) of a stressed magnetic field to an equilibrium (Russell et al 2015). Note that the rate of change of the helicity $H$ has long been established, at least when the reference field is potential (Berger and Field 1984, Finn and Antonsen 1985).

In this paper, we discuss these three problems in details in the simplest case where the field $\mathbf{B}$ has a simple topology and the part of $S$ where $B_{n}=0$ is a simple curve. In rough terms, this means that the magnetic lines of $\mathbf{B}$ simply connect in a continuous way an area of $S$ of positive polarity to an adjacent area of negative polarity by merely bridging above a polarity inversion line. This is certainly a significant restriction, but this will allow us to get interesting results without having to develop lengthy and heavy arguments that would certainly obscure some of the main issues. Question (i) above is discussed in section 3 and 28, where we relate $H$ and $h$ to the magnetic mapping of $\mathbf{B}$ (a mapping associating together the two footpoints of any magnetic line on $S$ ) and to the boundary values of a pair of Euler potentials of $\mathbf{B}$, respectively. The results so obtained are used in section 5 to deal with question (ii), which is shown to have a positive answer when the reference potential is of a specific type. They are also of much help in section 6 , where we consider question (iii) in the case where the boundary motions preserve $B_{n}$ on $S$. Under that assumption, we are able to establish a new formula for the time variation of the helicity of a moving line, which complements formulae previously obtained by Russell et al (2015). All our results are summarized in our concluding section 7, where we also announce some generalizations of them (to complex topology fields, to domains of different types, ...) that will be presented in a forthcoming paper.

\section{Assumptions and basic definitions}

\subsection{Assumptions}

Let $D$ be a simply connected domain of space bounded by the connected surface $S$, and denote as $\hat{\mathbf{n}}$ the external normal to $S$. For instance, $D$ may be either the (bounded) interior or the (unbounded) exterior of a sphere $S$, representing respectively the interior 
of a star and its corona, or a half-space, bounded by the plane $S$, representing a localized active region of the solar corona. $D$ is taken to contain a perfectly conducting plasma (this assumption will be given up in section 6) and a smooth magnetic field $\mathbf{B}$ that satisfies the following conditions:

- The parts $S^{+}$and $S^{-}$of $S$ of positive and negative polarity, respectively, are connected subdomains of $S$ that are in contact along a simple curve $S^{0}$ on which $B_{n}=0$. We remind the reader that, by definition, the magnetic lines emerge into $D$ through $S^{+}$, where then $-\mathbf{B} \cdot \hat{\mathbf{n}}=-B_{n}>0$, and leave $D$ through $S^{-}$, where then $-B_{n}<0$.

- $\mathbf{B}$ is line-tied, i.e., all its field lines (except possibly a negligible subset) are tied to the boundary $S$. Then (almost) any line connects a point $\mathbf{r}$ of $S^{+}$(we denote that line as $\mathcal{L}(\mathbf{r}))$ to a point $\mathbf{M}(\mathbf{r})$ of $S^{-}$. The function $\mathbf{M}: S^{+} \rightarrow S^{-}$defined that way is called the magnetic mapping.

- $\mathbf{B}$ is a simple topology field (STF), meaning that, in addition to be line-tied, it has a continuous magnetic mapping $\mathbf{M}$. The lines of $\mathbf{B}$ thus connect in a continuous way $S^{+}$to $S^{-}$by bridging over the curve $S^{0}$. To be an STF, B must not vanish in $D$ (no neutral points), nor have bald patches, i.e., lines that are tangent to $S$ at a point of $S^{0}$ (Titov et al 2002).

- If $D$ is unbounded, B decays sufficiently fast at infinity for all the integrals defined below to be convergent.

A B satisfying these assumptions will be said to be an admissible magnetic field.

\subsection{Definitions}

Consider an admissible field $\mathbf{B}$ and select an arbitrary field $\mathbf{B}_{r}$ (which may not be admissible) whose normal component on $S$ is the same as B's, i.e., $B_{r n}=B_{n}$ on $S$ (we also require that $\mathbf{B}_{r}$ decays sufficiently fast at infinity if $D$ is unbounded). Then the helicity of $\mathbf{B}$ relative to the reference field $\mathbf{B}_{r}$ is defined by (Berger and Field 1984, Finn and Antonsen 1985)

$$
H=H\left[\mathbf{B} / \mathbf{B}_{r}\right]=\int_{D}\left(\mathbf{A}+\mathbf{A}_{r}\right) \cdot\left(\mathbf{B}-\mathbf{B}_{\mathbf{r}}\right) \mathrm{d} v,
$$

where $\mathbf{A}$ and $\mathbf{A}_{r}$ are arbitrary vector potentials of $\mathbf{B}$ and $\mathbf{B}_{r}$, respectively. $H$ is gauge invariant, i.e., it keeps the same value under the gauge transforms $\mathbf{A} \rightarrow \mathbf{A}+\boldsymbol{\nabla} g$ and $\mathbf{A}_{r} \rightarrow \mathbf{A}_{r}+\nabla g_{r}$, where $g$ and $g_{r}$ are arbitrary functions. For short, we shall often refer to $H$ as the helicity of $\mathbf{B}$.

In most astrophysical applications, it is usual to choose the reference field to be the unique potential field $\mathbf{B}_{\pi}$ in $D$ such that $B_{\pi n}=B_{n}$ on $S$ (note that $\mathbf{B}_{\pi}$ may fail to be an STF). In that case, the quantity $H_{\mathrm{rel}}=H\left[\mathbf{B} / \mathbf{B}_{\pi}\right]$ is simply called the relative helicity of $\mathbf{B}$. It is important to note that $H_{\text {rel }}$ is an intrinsic property of $\mathbf{B}$ as $\mathbf{B}_{\pi}$ is fully determined from $\mathbf{B}$. This makes $H_{\text {rel }}$ most special among all the possible $H$. 
Let us now fix a particular vector potential of $\mathbf{B}_{r}, \mathbf{C}_{r}$ say, and denote as $\mathbf{C}$ a vector potential of $\mathbf{B}$ that satisfies the gauge condition (gc hereafter)

$$
\mathbf{C}_{s}=\mathbf{C}_{r s} \quad \text { on } S \text {, }
$$

where the subscript 's' indicates a vector component tangent to $S$. Then the field line helicity of $\mathbf{B}$ relative to $\mathbf{C}_{r}$ is defined by (Berger 1988)

$$
h(\mathbf{r})=h\left[\mathbf{B} / \mathbf{C}_{r} ; \mathbf{r}\right]=\int_{\mathcal{L}(\mathbf{r})} \mathbf{C} \cdot \mathrm{d} \boldsymbol{l}, \quad \mathbf{r} \in S^{+} .
$$

This quantity is invariant with respect to the gauge transforms of $\mathbf{C}$ that respect gc. Indeed, two vector potentials $\mathbf{C}$ and $\mathbf{C}^{\prime}$ satisfying gc differ by the gradient of a function $g$ that takes a constant value on $S$, and

$$
\int_{\mathcal{L}} \boldsymbol{\nabla} g \cdot \mathrm{d} \boldsymbol{l}=[g]_{\mathcal{L}}=0
$$

where we have introduced the notation $[X]_{\mathcal{L}(\mathbf{r})}=X(\mathbf{M}(\mathbf{r}))-X(\mathbf{r})$. Then $h$ is independent of the particular choice that is made for $\mathbf{C}$. It depends only on the choice of $\mathbf{C}_{r}$, which justifies our notation $h\left[\mathbf{B} / \mathbf{C}_{r} ; \mathbf{r}\right]$. Actually, it would have been sufficient to fix the value of $\mathbf{C}_{r s}$ on $S$, and we could have defined $h$ in a more general way, without making reference to the field $\mathbf{B}_{r}$. We have refrained to do so here to keep the presentation as simple as possible.

If we use the specific determinations $\mathbf{C}_{r}$ and $\mathbf{C}$ for the vector potentials in equation (1), $H$ takes the form

$$
H=\int_{D}\left(\mathbf{C} \cdot \mathbf{B}-\mathbf{C}_{r} \cdot \mathbf{B}_{r}\right) \mathrm{d} v=\int_{S^{+}} h\left(-B_{n}\right) \mathrm{d} s-H_{r},
$$

where we have set

$$
H_{r}=\int_{D} \mathbf{C}_{r} \cdot \mathbf{B}_{r} \mathrm{~d} v
$$

To compute the integral of the first term in the middle member of equation (5), we have decomposed the domain $D$ into elementary flux tubes $\mathrm{d} \mathcal{T}$ constructed about the magnetic lines $\mathcal{L}$ of $\mathbf{B}$ and written the volume element inside $\mathrm{d} \mathcal{T}$ in the form $\mathrm{d} v=\mathrm{d} s \mathrm{~d} l=\mathrm{d} \phi \mathrm{d} l / B$, with $\mathrm{d} l$ the length element along $\mathcal{L}, \mathrm{d} s$ the area of a normal cross-section of $\mathrm{d} \mathcal{T}$, and $\mathrm{d} \phi=B \mathrm{~d} s$ the constant flux inside $\mathrm{d} \mathcal{T}$. And we have noted finally that $\mathrm{d} \phi=-B_{n} \mathrm{~d} s$, where $\mathrm{d} s$ is now the area of the intersection of $\mathrm{d} \mathcal{T}$ with $S^{+}$. Note that we have isolated the term $\left(-B_{n}\right)$ in the integral appearing in the right-hand side of equation (5). This is just a way to keep in mind that this term is positive. Note also that $\mathbf{C}_{r}$ can always be selected in such a way that the convenient condition $H_{r}=0$ be satisfied (if $H_{r} \neq 0$, set $\mathbf{C}_{r}^{\prime}=\mathbf{C}_{r}+\lambda \nabla g$, with $g=B_{n}$ on $S$ and $\lambda=-H_{r} / \int_{S} B_{n}^{2} \mathrm{~d} s$; then $\left.H_{r}^{\prime}=0\right)$.

\subsection{Topological invariance}

We say that the field $\mathbf{B}^{\prime}$ is topologically equivalent to the field $\mathbf{B}$ - which we denote as $\mathbf{B}^{\prime} \sim \mathbf{B}$ - if there does exist a continuous ideal MHD deformation that transforms $\mathbf{B}$ 
into $\mathbf{B}^{\prime}$ without moving the footpoints of the lines on the boundary $S$. More explicitly, we have $\mathbf{B}^{\prime} \sim \mathbf{B}$ if we can find a velocity field $\mathbf{v}(\mathbf{r}, t)$ vanishing on $S^{+}$and $S^{-}$and a magnetic field $\mathbf{b}(\mathbf{r}, t)$, both defined in $D \times\{0 \leq t \leq T\}$, that are related to each other by the ideal MHD equation of evolution,

$$
\partial_{t} \mathbf{b}=\boldsymbol{\nabla} \times(\mathbf{v} \times \mathbf{b}),
$$

and are such that

$$
\mathbf{b}(t=0)=\mathbf{B} \text { and } \mathbf{b}(t=T)=\mathbf{B}^{\prime} .
$$

Quite obviously, two topologically equivalent fields have the same normal component on $S$ (by equation (7), the vanishing of $\mathbf{v}$ on $S$ implies that $b_{n}$ keeps a constant value all along the evolution, whence $B_{n}^{\prime}=b_{n}=B_{n}$ ) and it is quite clear that they have also the same magnetic mapping, $\mathbf{M}^{\prime}=\mathbf{m}=\mathbf{M}$.

A functional $X[\mathbf{B}]$ (which may be a number or a function) such that $X\left[\mathbf{B}^{\prime}\right]=X[\mathbf{B}]$ if $\mathbf{B}^{\prime} \sim \mathbf{B}$ is called an ideal MHD topological invariant. As it is well known, the helicity $H$ relative to $\mathbf{B}_{r}$ (a number) and the field line helicity $h(\mathbf{r})$ relative to $\mathbf{C}_{r}$ (a function) are both topological invariants. This can be shown by a direct calculation using equation (7) (see, respectively, Berger and Field 1984 and Berger 1988).

\section{Helicity and magnetic mapping}

In this section, we establish some new formulae that relate either the field line helicity $h$ or the helicity $H$ to the magnetic mapping $\mathbf{M}$.

\subsection{Magnetic mapping}

The magnetic mapping has already been defined in subsection 2.1. It is the one-to-one mapping $\mathbf{M}: S^{+} \rightarrow S^{-}$that associates to the positive footpoint position $\mathbf{r} \in S^{+}$of the magnetic line $\mathcal{L}(\mathbf{r})$ its negative footpoint position $\mathbf{M}(\mathbf{r}) \in S^{-}$, and it is taken here to be continuous (in fact we shall even assume that it is differentiable). Of course, $\mathbf{M}$ has an inverse mapping $\mathbf{M}^{-1}: S^{-} \rightarrow S^{+}$with the same properties, and we could as well use the latter in all the calculations below in place of $\mathbf{M}$.

It is often convenient to represent the magnetic mapping with the help of curvilinear coordinates defined on $S^{ \pm}$. Let us introduce such coordinates $x=\left(x^{1}, x^{2}\right)$ on $S^{+}$and $X=\left(X^{1}, X^{2}\right)$ on $S^{-}$. To them are associated, respectively, the basis $\left(\mathbf{e}_{1}, \mathbf{e}_{2}\right)$ and $\left(\mathbf{E}_{1}, \mathbf{E}_{2}\right)$ (with $\mathbf{e}_{j}=\partial \mathbf{r} / \partial x^{j}$ and $\left.\mathbf{E}_{j}=\partial \mathbf{r} / \partial X^{j}\right)$, and the dual basis $\left(\boldsymbol{\nabla}_{s} x^{1}, \boldsymbol{\nabla}_{s} x^{2}\right)$ and

$\left(\boldsymbol{\nabla}_{s} X^{1}, \boldsymbol{\nabla}_{s} X^{2}\right.$ ) (we remind the reader that $\hat{\mathbf{e}}_{i} \cdot \boldsymbol{\nabla}_{s} x^{j}=\hat{\mathbf{E}}_{i} \cdot \boldsymbol{\nabla}_{s} X^{j}=\delta_{i}^{j}$, where $\delta_{i}^{j}$ is the usual Kronecker symbol). The magnetic mapping can then be expressed as

$$
\mathbf{M}: x=\left(x^{1}, x^{2}\right) \rightarrow X=X(x)=\left(X^{1}\left(x^{1}, x^{2}\right), X^{2}\left(x^{1}, x^{2}\right)\right),
$$

and its gradient (or in more sophisticated terms, avoided here, its tangent mapping) can be written in the form

$$
\boldsymbol{\nabla}_{s} \mathbf{M}(x)=\partial_{j} X^{k}(x) \boldsymbol{\nabla}_{s} x^{j}(x) \otimes \mathbf{E}_{k}(X(x))
$$


(here $\partial_{j} X^{k}=\partial X^{k} / \partial x^{j}$; note our convention for the order of the indices). Multiplying $\boldsymbol{\nabla}_{s} \mathbf{M}$ on the left by the vector $\mathbf{Z}=Z^{l} \mathbf{e}_{l}$ tangent to $S^{+}$at $\mathbf{r}$ produces the vector $Z^{j} \partial_{j} X^{k} \mathbf{E}_{k}$ tangent to $S^{-}$at $\mathbf{M}(\mathbf{r})$, while multiplying it on the right by the vector $\mathbf{Z}=Z_{l} \boldsymbol{\nabla}_{s} X^{l}$ tangent to $S^{-}$at $\mathbf{M}(\mathbf{r})$ produces the vector $\partial_{j} X^{k} Z_{k} \boldsymbol{\nabla}_{s} x^{j}$ tangent to $S^{+}$ at $\mathbf{r}$.

As noted in section 2.3, we have $\mathbf{M}=\mathbf{M}^{\prime}$ if $\mathbf{B} \sim \mathbf{B}^{\prime}$. The converse is true for admissible fields: their topology is fully characterized by their magnetic mapping, i.e., we have $\mathbf{B} \sim \mathbf{B}^{\prime}$ if $\mathbf{M}=\mathbf{M}^{\prime}$ (Zweibel and Li 1987).

\subsection{Field line helicity}

Consider a magnetic line $\mathcal{L}(\mathbf{r})$ of the admissible field $\mathbf{B}$ and an arbitrary simple oriented curve $\mathcal{C}(\mathbf{r})$ of $S$ that connects $\mathbf{r}$ to $\mathbf{M}(\mathbf{r})$. Applying Stokes theorem to the vector potential $\mathrm{C}$ and the closed contour $\mathcal{L} \cup-\mathcal{C}$, we obtain at the point $\mathbf{r}$

$$
h=\Phi+\int_{\mathcal{C}} \mathbf{C}_{r s} \cdot d \mathbf{l},
$$

where we have used that $\mathbf{C} \cdot d \mathbf{l}=\mathbf{C}_{r s} \cdot d \mathbf{l}$ on $S$ as a consequence of gc and denoted as $\Phi$ the magnetic flux through $\mathcal{L} \cup-\mathcal{C}$ (this is the flux through an arbitrary surface $\sigma$ of $D$ bounded by that contour). If we deform $\mathbf{B}$ into a topologically equivalent field $\mathbf{B}^{\prime}$, equation (11) applied to the latter field has the same second term in the right-hand side (as we do not move the footpoints on $S$, we can use the same curve $\mathcal{C}$ ) and the flux $\Phi^{\prime}$ through the transformed contour $\mathcal{L}^{\prime} \cup-\mathcal{C}$ is equal to $\Phi$ by the frozen flux theorem. Then $h^{\prime}=h$, i.e., we recover immediately the fact that field line helicity is an ideal MHD topological invariant.

Let us now choose for $\mathcal{C}$ the simple curve $\overline{\mathcal{C}}(\mathbf{r})=\mathcal{C}\left(\mathbf{r}, \mathbf{r}_{0}\right) \cup-\mathbf{M}\left(\mathcal{C}\left(\mathbf{r}, \mathbf{r}_{0}\right)\right)$, where $\mathcal{C}\left(\mathbf{r}, \mathbf{r}_{0}\right)$ is a curve of $S^{+}$that connects $\mathbf{r}$ to some point $\mathbf{r}_{0}$ of $S^{0} . \mathbf{M}\left(\mathcal{C}\left(\mathbf{r}, \mathbf{r}_{0}\right)\right)$ is its image by the magnetic mapping and it connects on $S^{-}$the point $\mathbf{M}(\mathbf{r})$ to $\mathbf{r}_{0}$. Then $\Phi=0$ (just choose $\sigma$ to be the magnetic surface made of all the magnetic lines originating from $\left.\mathcal{C}\left(\mathbf{r}, \mathbf{r}_{0}\right)\right)$, and we get from equation (11)

$$
h=\int_{\overline{\mathcal{C}}} \mathbf{C}_{r s} \cdot \mathrm{d} \boldsymbol{l} .
$$

This formula exhibits the topological invariance of $h$ in a quite striking way.

By decomposing the integral in the right-hand side of equation (12) into an integral

along $\mathcal{C}\left(\mathbf{r}, \mathbf{r}_{0}\right)$ and one along $-\mathbf{M}\left(\mathcal{C}\left(\mathbf{r}, \mathbf{r}_{0}\right)\right)$, and by making in the latter the change of variable defined by the magnetic mapping, we obtain the equivalent expression

$$
\begin{aligned}
h(\mathbf{r}) & =\int_{\mathcal{C}\left(\mathbf{r}, \mathbf{r}_{0}\right)} \mathbf{C}_{r s} \cdot \mathrm{d} \boldsymbol{l}+\int_{-\mathbf{M}\left[\mathcal{C}\left(\mathbf{r}, \mathbf{r}_{0}\right)\right]} \mathbf{C}_{r s} \cdot \mathrm{d} \boldsymbol{l} \\
& =\int_{\mathcal{C}\left(\mathbf{r}, \mathbf{r}_{0}\right)}\left(\mathbf{C}_{r s}-\nabla_{s} \mathbf{M} \cdot \widetilde{\mathbf{C}_{r s}}\right) \cdot \mathrm{d} \boldsymbol{l} .
\end{aligned}
$$

We have introduced here the notation $\widetilde{X}(\mathbf{r})=X(\mathbf{M}(\mathbf{r}))$. If we use coordinates, equation 
(13) assumes the form

$$
h\left(x^{1}, x^{2}\right)=\int_{\mathcal{C}\left(\mathbf{r}, \mathbf{r}_{0}\right)}\left(C_{r j}-\partial_{j} X^{k} \widetilde{C_{r k}}\right) \mathrm{d} l^{j} .
$$

It is obtained with help from equation (10) and the decompositions $\mathbf{C}_{r s}=C_{r i} \boldsymbol{\nabla}_{s} x^{i}$ and $\mathrm{d} \boldsymbol{l}=d l^{j} \mathbf{e}_{j}$ on $S^{+}$, and $\mathbf{C}_{r s}=C_{r i} \boldsymbol{\nabla}_{s} X^{i}$ on $S^{-}$.

Finally we note that taking the surface gradient of equation (13) leads to the formula (useful below)

$$
\nabla_{s} h=\nabla_{s} \mathbf{M} \cdot \widetilde{\mathbf{C}_{r s}}-\mathbf{C}_{r s} .
$$

A similar relation was previously obtained in a particular geometrical setting by Aly (2014) and by Yeates and Hornig (2014; see their Lemma 2, where the relation is expressed in the language of differential forms).

\subsection{Helicity}

Using equation (13) in equation (5) leads to

$$
H=\int_{S^{+}}\left(\int_{\mathcal{C}\left(\mathbf{r}, \mathbf{r}_{0}\right)}\left(\mathbf{C}_{r s}-\nabla_{s} \mathbf{M} \cdot \widetilde{\mathbf{C}_{r s}}\right) \cdot \mathrm{d} \boldsymbol{l}\right)\left(-B_{n}\right) \mathrm{d} s-H_{r} .
$$

Another formula for $H$ can be obtained by transforming equation (5) with help from a standard formula of vector analysis, Stokes theorem, and the obvious fact that $h=0$ on $\partial S^{+}=S^{0}$. One obtains

$$
\begin{aligned}
H+H_{r} & =-\int_{S^{+}} h \boldsymbol{\nabla} \times \mathbf{C}_{r} \cdot \hat{\mathbf{n}} \mathrm{d} s \\
& =\int_{S^{+}}\left(\boldsymbol{\nabla}_{s} h \times \mathbf{C}_{r}\right) \cdot \hat{\mathbf{n}} \mathrm{d} s-\int_{\partial S^{+}} h \mathbf{C}_{r} \cdot \mathrm{d} \boldsymbol{l} \\
& =\int_{S^{+}}\left(\nabla_{s} h \times \mathbf{C}_{r s}\right) \cdot \hat{\mathbf{n}} \mathrm{d} s,
\end{aligned}
$$

whence

$$
H=\int_{S^{+}}\left[\left(\nabla_{s} \mathbf{M} \cdot \widetilde{\mathbf{C}_{r s}}\right) \times \mathbf{C}_{r s}\right] \cdot \hat{\mathbf{n}} \mathrm{d} s-H_{r}
$$

by using equation (15). In terms of the coordinates $\left(x^{1}, x^{2}\right)$ (which take their values in the domain $\mathcal{S}^{+}$and are chosen such that $\mathbf{e}_{1} \times \mathbf{e}_{2} \cdot \hat{\mathbf{n}}>0$ ), the last formula assumes the form

$$
H=\int_{\mathcal{S}^{+}} \varepsilon^{k j} \partial_{k} X^{i} \widetilde{C_{r i}} C_{r j} \mathrm{~d} x^{1} \mathrm{~d} x^{2}-H_{r}
$$

(the symbol $\varepsilon$ is defined by $\varepsilon^{11}=\varepsilon^{22}=0$ and $\varepsilon^{12}=-\varepsilon^{21}=1$ ).

The only quantities that intervene in the right-hand sides of equations (16), (18), and (19), are the tangential component of $\mathbf{C}_{r}$ on $S$ and the magnetic mapping $\mathbf{M}$. It is then obvious from any of these new formulae that two topologically equivalent fields $\mathbf{B}$ and $\mathbf{B}^{\prime}$ have the same helicity relative to $\mathbf{B}_{r}$ : they have indeed the same magnetic mapping and we can use the same vector potential $\mathbf{C}_{r}$ for computing $H$ and $H^{\prime}$. 


\section{Helicity and Euler potentials}

We next present expressions of $h$ and $H$ that are obtained by using an Euler potential representation of B (Stern 1970).

\subsection{Euler potentials}

A global representation of an admissible field $\mathbf{B}$ in terms of a pair $(U, V)$ of Euler potentials can be constructed as follows. We first choose in $S^{+}$a family of simple curves $\mathcal{C}_{u}$ with the following properties: (i) there is a unique $\mathcal{C}_{u}$ passing through any point of $S^{+}$(the curves cover $S^{+}$); (ii) each curve $\mathcal{C}_{u}$ connects together two points of the boundary $\partial S^{+}$; (iii) the label $u$ is the value of a function $U_{0}(\mathbf{r})$ satisfying $\nabla_{s} U_{0} \neq 0$, i.e., $\mathcal{C}_{u}=\left\{\mathbf{r} \in S^{+} \mid U_{0}(\mathbf{r})=u\right\}$. We orient each curve $\mathcal{C}_{u}$ by the tangent vector $\boldsymbol{\nabla}_{s} U_{0} \times \hat{\mathbf{n}}$, and we define along it an increasing arc-length $l$ that vanishes at the crossing with some curve $\Gamma$ that intersects transversally each $\mathcal{C}_{u}$. Next we set

$$
V_{0}(\mathbf{r})=-\int_{0}^{l(\mathbf{r})} \frac{B_{n}}{\left|\nabla_{s} U_{0}\right|} \mathrm{d} l,
$$

where the integral is computed along $\mathcal{C}_{u}=\mathcal{C}_{U_{0}(\mathbf{r})}$. The function $V_{0}$ is such that $B_{n}=$ $\left(\boldsymbol{\nabla}_{s} U_{0} \times \boldsymbol{\nabla}_{s} V_{0}\right) \cdot \hat{\mathbf{n}}$ and it vanishes on $\Gamma$. Clearly, a contour level $\mathcal{C}_{v}=\left\{\mathbf{r} \in S^{+} \mid V_{0}(\mathbf{r})=v\right\}$ of $V_{0}$ cuts at most once a contour level of $U_{0}$ (as $V_{0}$ increases along $\mathcal{C}_{u}$ ), and a connected component of $\mathcal{C}_{v}$ joins together two points of $\partial S^{+}$.

Next we define two functions $U$ and $V$ in $D$ by imposing the two following conditions: (i) $U$ and $V$ keep constant values along any magnetic line, i.e., they satisfy B $\cdot \nabla U=0$ and $\mathbf{B} \cdot \nabla V=0$ in $D$; (ii) $U$ and $V$ match on $S^{+}$the functions $U_{0}$ and $V_{0}$ previously constructed. We thus have, on the one hand $\mathbf{B}=\lambda \nabla U \times \nabla V$ in $D$, with the function $\lambda$ staying constant along any line as $\boldsymbol{\nabla} \cdot \mathbf{B}=(\boldsymbol{\nabla} U \times \boldsymbol{\nabla V}) \cdot \boldsymbol{\nabla} \lambda=\mathbf{B} \cdot \boldsymbol{\nabla} \lambda / \lambda=0$, and on the other hand $\lambda=1$ on $S^{+}$where $B_{n}=\lambda\left(\boldsymbol{\nabla}_{s} U_{0} \times \boldsymbol{\nabla}_{s} V_{0}\right) \cdot \hat{\mathbf{n}}=\left(\boldsymbol{\nabla}_{s} U_{0} \times \boldsymbol{\nabla}_{s} V_{0}\right) \cdot \hat{\mathbf{n}}$. Hence $\lambda=1$ in the whole $D$ and

$$
\mathbf{B}=\boldsymbol{\nabla} U \times \nabla V=\boldsymbol{\nabla} \times(U \nabla V) \text { in } D,
$$

showing that $\mathbf{B}$ admits the particular vector potential

$$
\mathbf{A}=U \nabla V .
$$

By construction, the Euler potentials are such that

$$
U(\mathbf{r})=\widetilde{U}(\mathbf{r}) \quad \text { and } \quad V(\mathbf{r})=\widetilde{V}(\mathbf{r}),
$$

i.e., they assume the same value at both footpoints of any line $\mathcal{L}(\mathbf{r})$. Then they do convey information on the connectivity of $\mathbf{B}$, which makes them particularly well adapted to the problems considered in this paper.

For further reference, we note that it is also possible to construct a pair $(U, V)$ by choosing on $S^{+}$a covering set $\left\{\mathcal{C}_{u}\right\}$ made of closed nested curves (Aly 1990). In that case, we take the parameter $u$ to be the magnetic flux through $\mathcal{C}_{u}$. The second potential can still be constructed on $S^{+}$by using equation (20), but it now exhibits a 
discontinuity on $\Gamma$ (on which $l=0$ ). Of course, this discontinuity extends into $D$, where there is a singular surface with $V=0$ on one side and $V=1$ on the other side. The vector potential $\mathbf{A}=U \boldsymbol{\nabla} V$ stays continuous in the whole $D$.

\subsection{Field line helicity}

We require here the selected reference field $\mathbf{B}_{r}$ to be admissible. This allows us introducing the Euler representations $\mathbf{B}=\nabla U \times \nabla V$ and $\mathbf{B}_{r}=\nabla U_{r} \times \nabla V_{r}$, and the particular vector potentials $\mathbf{C}$ and $\mathbf{C}_{r}$ can be written in the form

$$
\mathbf{C}=U \nabla V+\nabla f \quad \text { and } \quad \mathbf{C}_{r}=U_{r} \nabla V_{r}+\nabla f_{r}
$$

for some functions $f$ and $f_{r}$. Hence

$$
h(\mathbf{r})=[f]_{\mathcal{L}(\mathbf{r})}=\tilde{f}(\mathbf{r})-f(\mathbf{r}) \quad \text { for } \mathbf{r} \in S^{+},
$$

with $f$ determined by (boundary condition gc)

$$
U \nabla_{s} V+\nabla_{s} f=U_{r} \nabla_{s} V_{r}+\nabla_{s} f_{r} \quad \text { on } S .
$$

For any two admissible fields $\mathbf{B}$ and $\mathbf{B}^{\prime}$ with the same normal component on $S$, we can construct their Euler potentials in such a way that $U=U^{\prime}$ on $S^{+}$, in which case we also have on that surface $V=V^{\prime}$ by equation (20) and $f=f^{\prime}$ by equation (26) (taking the unessential integration constant equal to zero). Euler potentials $(U, V)$ and $\left(U^{\prime}, V^{\prime}\right)$ satisfying this constraint will be said to be "concordant". If the two fields are topologically equivalent, $\mathbf{B} \sim \mathbf{B}^{\prime}$, we have also $U=U^{\prime}, V=V^{\prime}$ and $f=f^{\prime}$ on $S^{-}$by equations (23) and (26), and then $h=h^{\prime}$ by equation (25): the topological invariance of $h$ is thus immediately recovered without further calculations. Finally, we remark that with the choice of concordant Euler potentials for $\mathbf{B}$ and $\mathbf{B}_{r}$, we have $h(\mathbf{r})=\widetilde{f}(\mathbf{r})-f_{r}(\mathbf{r})$, and we need to compute $f$ only on $S^{-}$.

\subsection{Helicity}

The helicity of $\mathbf{B}$ relative to the reference field $\mathbf{B}_{r}$ can be most easily obtained by a direct calculation starting from the definition (1). With $\mathbf{A}=U \boldsymbol{\nabla} V$ and $\mathbf{A}_{r}=U_{r} \boldsymbol{\nabla} V_{r}$, one gets by using a standard formula of vector analysis

$$
\begin{aligned}
H & =\int_{D}\left(U \nabla V+U_{r} \boldsymbol{\nabla} V_{r}\right) \cdot\left(\boldsymbol{\nabla} U \times \boldsymbol{\nabla} V-\boldsymbol{\nabla} U_{r} \times \nabla V_{r}\right) \mathrm{d} v \\
& =\int_{D}\left[U_{r} \boldsymbol{\nabla} V_{r} \cdot \boldsymbol{\nabla} \times(U \boldsymbol{\nabla} V)-U \nabla V \cdot \boldsymbol{\nabla} \times\left(U_{r} \boldsymbol{\nabla} V_{r}\right)\right] \mathrm{d} v \\
& =\int_{D} \boldsymbol{\nabla} \cdot\left(U \boldsymbol{\nabla} V \times U_{r} \boldsymbol{\nabla} V_{r}\right) \mathrm{d} v,
\end{aligned}
$$

and one obtains finally with help from Gauss theorem

$$
H=\int_{S} U U_{r}\left(\nabla_{s} V \times \nabla_{s} V_{r}\right) \cdot \hat{\mathbf{n}} \mathrm{d} s .
$$


This relation also holds when the Euler potentials are constructed by the alternative method sketched at the end of subsection 4.1 (Aly 1990). If we choose concordant Euler potentials for $\mathbf{B}$ and $\mathbf{B}_{r}$, the integral over $S$ reduces to an integral over $S^{-}$.

Equation (28) exhibits explicitly the topological invariance of $H$ as $U=U^{\prime}$ and $V=V^{\prime}$ on $S$ if we choose concordant Euler potentials for the two topologically equivalent fields $\mathbf{B}$ and $\mathbf{B}^{\prime}$.

\section{Does field line helicity characterize the topology of B?}

Under our assumptions, we know that two admissible fields $\mathbf{B}$ and $\mathbf{B}^{\prime}$ that are topologically equivalent have the same field line helicity relative to the vector potential $\mathbf{C}_{r}$. It seems natural to address the problem of the validity of the converse statement (Yeates and Hornig 2014): if for a given $\mathbf{C}_{r}$ one has $h=h^{\prime}$ for two admissible fields $\mathbf{B}$ and $\mathbf{B}^{\prime}$ with $B_{n}=B_{n}^{\prime}$ on $S$, are these two fields topologically equivalent? Here we are going to show that the answer to that question is positive if we choose a vector potential of reference of the form

$$
\mathbf{C}_{r}=U_{r} \boldsymbol{\nabla} V_{r}
$$

(i.e., $\left.f_{r}=0\right)$, with the pair of Euler potentials $\left(U_{r}, V_{r}\right)$ being computed by the method of subsection 4.1 .

Let us consider two admissible fields $\mathbf{B}$ and $\mathbf{B}^{\prime}$ that have the same normal component on $S$ and the same field line helicity relative to the vector potential of reference (29). As $h=h^{\prime}$, equation (15) implies

$$
\nabla_{s} h=\nabla_{s} \mathbf{M} \cdot \widetilde{\mathbf{C}_{r s}}-\mathbf{C}_{r s}=\nabla_{s} \mathbf{M}^{\prime} \cdot \widetilde{\mathbf{C}_{r s}}-\mathbf{C}_{r s}=\nabla_{s} h^{\prime} \quad \text { on } S^{+},
$$

where we have used the notation $\widetilde{X}^{\prime}(\mathbf{r})=X\left(\mathbf{M}^{\prime}(\mathbf{r})\right)$. With our choice for $\mathbf{C}_{r}$, we thus have

$$
\widetilde{U_{r}} \nabla_{s} \mathbf{M} \cdot \widetilde{\nabla_{s} V_{r}}=\widetilde{U_{r}^{\prime}} \nabla_{s} \mathbf{M}^{\prime} \cdot{\widetilde{\nabla_{s} V_{r}^{\prime}}}^{\prime} \text { on } S^{+}
$$

On the other hand, it is an immediate consequence of the chain rule that $\boldsymbol{\nabla}_{s} \mathbf{M} \cdot \widetilde{\nabla_{s} F}=$ $\nabla_{s} \widetilde{F}$ on $S^{+}$for any function $F$ defined on $S$, whence

$$
\widetilde{U_{r}} \nabla_{s} \widetilde{V}_{r}={\widetilde{U_{r}}}^{\prime} \nabla_{s} \widetilde{V}_{r}^{\prime} \quad \text { on } S^{+} \text {. }
$$

The gradients of $\widetilde{V}_{r}$ and $\widetilde{V}_{r}^{\prime}$ being collinear on $S^{+}, \widetilde{V}_{r}^{\prime}$ needs to assume a constant value along any connected component of a contour level of $\widetilde{V}_{r}$. But as seen in subsection 4.1 , each such curve cuts the boundary $\partial S^{+}$, along which $\widetilde{V}_{r}^{\prime}=\widetilde{V}_{r}=V_{r}$. Therefore we have $\widetilde{V}_{r}^{\prime}=\widetilde{V}_{r}$ in the whole $S^{+}$, and also $\widetilde{U_{r}}{ }^{\prime}=\widetilde{U_{r}}$ by equation (32). Clearly, this implies that $\mathbf{M}^{\prime}=\mathbf{M}$, and the two fields $\mathbf{B}$ and $\mathbf{B}^{\prime}$ are indeed topologically equivalent.

We can conclude that the topology of an admissible field, which is characterized by its $2 \mathrm{D}$ magnetic mapping $\mathbf{M}$, can be encoded as well in the two scalar quantities $B_{n}$ and $h$. 


\section{Field line helicity evolution in presence of boundary motions and/or dissipative effects}

Up to now, we have considered deformations of a magnetic field that were constrained by the frozen-in law and that kept fixed the positions of the footpoints on $S$. We consider

in this section a more general situation where the plasma on the boundary $S$ is allowed to move and/or there are non-ideal processes acting in $D$.

\subsection{Assumptions and reminder}

We make here the following assumptions:

- At least one of the following items holds true

- There are non-ideal processes at work in $D$ and they are described by the unspecified term $\mathbf{N}$ in Ohm's law

$$
\mathbf{E}+\mathbf{v} \times \mathbf{B} / c=\mathbf{N} .
$$

Such a term is present, e.g., in the standard resistive MHD model, where we have $\mathbf{N}=\eta \mathbf{j}$, with $\eta$ the magnetic diffusivity and $\mathbf{j}$ the electric current density. We require $\mathbf{N}$ to vanish in a neighborhood of the perfectly conducting boundary $S$. For instance, the non-ideal processes may be localized in small parts of $D$, as in the theory of 3D reconnection developed by Schindler and coworkers (Schindler 2006, and references therein).

- The plasma on $S$ moves at the prescribed velocity $\mathbf{v}$. In order to simplify the presentation, we choose $\mathbf{v}$ to be tangential to $S$, i.e., $\mathbf{v}=\mathbf{v}_{s}$ (this restriction can be easily given up).

- The initial magnetic field is admissible, with its lines thus connecting in a continuous way the parts $S^{+}$and $S^{-}$of $S$, and this property is preserved during the evolution of the field even when $\mathbf{N} \not \equiv 0$. In particular, the non-ideal processes do not create inside $D$ regions that are magnetically disconnected from $S$.

As it is well known (see, e.g., Schindler 2006), it is possible to write $\mathbf{N}$ in the form

$$
\mathbf{N}=-\frac{\mathbf{u} \times \mathbf{B}}{c}+\boldsymbol{\nabla} \psi,
$$

where

$$
\begin{aligned}
& \psi(\mathbf{r})=\psi_{0}(\underline{\mathbf{r}})+\int_{\mathcal{L}(\underline{\mathbf{r}, \mathbf{r})}} \mathbf{N} \cdot \mathrm{d} \boldsymbol{l}, \\
& \mathbf{u}(\mathbf{r})=c \frac{\mathbf{B} \times(\boldsymbol{\nabla} \psi-\mathbf{N})}{B^{2}}+\lambda \mathbf{B} .
\end{aligned}
$$

In these equations, $\psi_{0}$ is an arbitrary function defined on $S^{+}, \underline{\mathbf{r}}$ is the position of the footpoint on $S^{+}$of the line passing through $\mathbf{r}, \mathcal{L}(\underline{\mathbf{r}}, \mathbf{r})$ denotes the part of $\mathcal{L}(\underline{\mathbf{r}})$ comprised between $\underline{\mathbf{r}}$ and $\mathbf{r}$, and $\lambda$ is an arbitrary function defined in $D$ (time dependence is 
understood). Here, we choose $\lambda$ in such a way that $u_{n}=\hat{\mathbf{n}} \cdot \mathbf{u}=0$ (and then $\mathbf{u}=\mathbf{u}_{s}$ ) on $S$. Setting

$$
\mathbf{w}=\mathbf{v}+\mathbf{u}
$$

and using equations (33)-(36), one gets

$$
\mathbf{E}+\frac{\mathbf{w} \times \mathbf{B}}{c}=\nabla \psi
$$

Curling that equation and taking Faraday's induction law into account leads to

$$
\frac{\partial \mathbf{B}}{\partial t}=\nabla \times(\mathbf{w} \times \mathbf{B})
$$

which implies that the velocity $\mathbf{w}$ is both flux-preserving and line-preserving. The meaning of $\mathbf{u}$ is clear: it represents the velocity of the line passing through a point $\mathbf{r}$ with respect to the element of plasma located at that point.

Our aim now is to compute the time derivative of the helicity $h(t)$ of a particular magnetic line $\mathcal{L}(t)=\mathcal{L}(\mathbf{r}(t), t)$ that moves in $D$ with the velocity $\mathbf{w}$. As $\mathbf{w}=\mathbf{v}+\mathbf{u}=$ $\mathbf{v}_{s}+\mathbf{u}_{s}=\mathbf{w}_{s}$ on $S$ by our assumptions above, $\mathbf{w}_{s}$ represents the velocity of the footpoints of $\mathcal{L}(t)$ located at $\mathbf{r}(t)$ and $\mathbf{M}(\mathbf{r}(t), t)$. Two remarks are in order at this point:

- For computing $h(t)$, we have to fix the reference field, $\mathbf{B}_{r}$, and its vector potential, $\mathbf{C}_{r}$, at each time $t$. One then has to face a serious problem of interpretation: what does it mean to compare the values of $h$ at two different times (the same problem arises for $H$, except in the case where the potential field is chosen as the reference field). If we impose indeed an arbitrary evolution to $\mathbf{B}_{r}$ and $\mathbf{C}_{r}$, then it is clear that the evolution of $h(t)$ will not have any sensible meaning (for instance, we can obtain a changing $h$ even if $\mathbf{B}$ and $\mathbf{B}_{r}$ stay constant by just performing a timedependent gauge transform on $\mathbf{C}_{r}$ ). For each specific problem we have to deal with, it is thus necessary to fix some physically meaningful rules of selection for $\mathbf{B}_{r}$ and $\mathbf{C}_{r}$ (see, e.g., Yeates and Hornig (2016) for an exemple). We shall not discuss this problem here, as we are mainly interested in the formal properties of $h$. We shall content ourselves to make below a choice that seems quite natural in view of our specific assumptions. It may be worth noticing that a similar problem of interpretation arises when one wants to introduce a local flux of relative magnetic helicity through the boundary $S$ as there is no gauge-invariant definition of such a quantity. Physical considerations, however, allow to select an appropriate gauge making the concept quite useful in solar physics (Démoulin and Pariat 2009).

- The unspecified function $\psi_{0}$ appearing in equation (35) induces a well known arbitrariness in the definition of $\mathbf{w}$. When $\mathbf{N} \equiv 0$, one chooses most generally $\psi_{0}=0$, which implies $\mathbf{w}_{\perp}=\mathbf{v}_{\perp}$ (the subscript $\perp$ indicating a component normal to $\mathbf{B})$, i.e., the standard frozen-in law. This choice may be also convenient in the case where $\mathbf{N} \not \equiv 0$ and we shall adopt it below as it still allows defining $\mathcal{L}(t)$ in a unique and physically clear way. 


\subsection{Time evolution of field line helicity in a specific case}

To keep the presentation as simple as possible, we make here the following specific assumptions:

- The motion of the plasma on the boundary $S$ preserves $B_{n}$, which implies the existence of a function $\zeta$ defined on $S$ such that

$$
B_{n} \mathbf{v}=B_{n} \mathbf{v}_{s}=\hat{\mathbf{n}} \times \nabla_{s} \zeta
$$

This choice allows us to choose time-independent $\mathbf{B}_{r}$ and $\mathbf{C}_{r}$, which may be thought to give a clear physical meaning to the evolving line helicity $h(t)$.

- The reference vector potential is chosen to be of the form $\mathbf{C}_{r}=U_{r} \boldsymbol{\nabla} V_{r}$, with $\left(U_{r}, V_{r}\right)$ a pair of time independent Euler potentials of $\mathbf{B}_{r}$ constructed by the method of section 4.1. We also introduce (time-dependent) Euler potentials $U$ and $V$ for the evolving field $\mathbf{B}$. They are constructed at the initial time in the same way as $\left(U_{r}, V_{r}\right)$ (with which they may be concordant or not), and are determined at any later time by

$$
\frac{\mathrm{d} U}{\mathrm{~d} t}=0 \text { and } \frac{\mathrm{dV}}{\mathrm{dt}}=0, \text { where } \frac{\mathrm{d}}{\mathrm{dt}}=\frac{\partial}{\partial \mathrm{t}}+\mathbf{w} \cdot \nabla,
$$

i.e., we require that they keep constant values when one follows the motion of any field line. As it is well-known, such a method for computing $U$ and $V$ is compatible with equation (39) (this equation is automatically satisfied by $\mathbf{B}=\boldsymbol{\nabla} U \times \nabla V$ if $U$ and $V$ are solutions of equation (41)). The vector potential of $\mathbf{B}$ satisfying the gauge condition gc can then be written $\mathbf{C}=U \boldsymbol{\nabla} V+\boldsymbol{\nabla} f$, with $f$ determined on $S$ by

$$
U_{r} \nabla_{s} V_{r}=U \nabla_{s} V+\nabla_{s} f
$$

(this is just equation (26) with $f_{r}=0$ ).

- Using equation (34) on $S$, where $\mathbf{N}=0$ and $u_{n}=0$, we obtain after crossing with $\hat{\mathbf{n}}$

$$
(\mathbf{u} \times \mathbf{B}) \times \hat{\mathbf{n}}=-B_{n} \mathbf{u}_{s}=\nabla_{s}(c \psi) \times \hat{\mathbf{n}},
$$

and combining this result with equations (37) and (40) leads to

$$
B_{n} \mathbf{w}_{s}=\hat{\mathbf{n}} \times \nabla_{s}(\zeta+c \psi) .
$$

- We choose the arbitrary function $\psi_{0}=0$, implying that the potential $\psi$ assumes the boundary values $\psi=0$ on $S^{+}$and

$$
\psi=\mathcal{N}=\int_{\mathcal{L}(t)} \mathbf{N} \cdot \mathrm{d} \boldsymbol{l} \text { on } S^{-} .
$$

Therefore, we have $\mathbf{u}_{s}=0$ on $S^{+}$, and a line $\mathcal{L}(t)$ is always connected to the same element of plasma moving on $S^{+}$. This provides a clear physical identification of $\mathcal{L}(t)$. On $S^{-}$on the contrary, the footpoint of $\mathcal{L}(t)$ drifts in general with respect to the plasma once that line goes through a non-ideal part of $D($ where $\mathbf{N} \neq 0$ ). 
To compute the time variation of $h(t)$, we first differentiate equation (42) with respect to time at a fixed point $\mathbf{r}$ of $S$, which results in

$$
\begin{aligned}
0 & =\frac{\partial U}{\partial t} \boldsymbol{\nabla}_{s} V-\frac{\partial V}{\partial t} \boldsymbol{\nabla}_{s} U+\boldsymbol{\nabla}_{s}\left(U \frac{\partial V}{\partial t}+\frac{\partial f}{\partial t}\right) \\
& =\mathbf{w}_{s} \times\left(\boldsymbol{\nabla}_{s} U \times \boldsymbol{\nabla}_{s} V\right)+\boldsymbol{\nabla}_{s}\left(-U \mathbf{w} \cdot \boldsymbol{\nabla}_{s} V+\frac{\partial f}{\partial t}\right) \\
& =\nabla_{s}\left(\frac{\partial f}{\partial t}-U \mathbf{w} \cdot \nabla_{s} V+\zeta+c \psi\right) .
\end{aligned}
$$

Here we have used equation (41) to get the second line and equation (44) and the relation $\boldsymbol{\nabla}_{s} U \times \boldsymbol{\nabla}_{s} V=B_{n} \hat{\mathbf{n}}$ to get the third line. The quantity in parentheses in the last line can then be taken to vanish (a time-dependent "constant" of integration can be absorbed by redefining $f$ ), and we can write

$$
\frac{\mathrm{d} f}{\mathrm{~d} t}=\frac{\partial f}{\partial t}+\mathbf{w} \cdot \nabla_{s} f=U_{r} \mathbf{w} \cdot \nabla_{s} V_{r}-(\zeta+c \psi),
$$

where we have used equation (42) to eliminate $\nabla_{s} f$. On the other hand, using $U_{r}$ and $V_{r}$ as coordinates on both $S^{+}$and $S^{-}$, we have $(\zeta+c \psi)=(\zeta+c \psi)\left(U_{r}, V_{r}, t\right)$ (with the functional dependence being of course different on $S^{+}$and on $S^{-}$). Whence

$$
U_{r} \mathbf{w} \cdot \nabla_{s} V_{r}=U_{r} \frac{\partial}{\partial U_{r}}(\zeta+c \psi) \hat{\mathbf{n}} \cdot\left(\nabla_{s} U_{r} \times \nabla_{s} V_{r}\right) / B_{n}
$$

with help from equation (44) and

$$
\frac{\mathrm{d} f}{\mathrm{~d} t}=U_{r} \frac{\partial}{\partial U_{r}}(\zeta+c \psi)-(\zeta+c \psi) \quad \text { on } \mathrm{S}^{+} \text {and on } \mathrm{S}^{-} .
$$

Finally, we have just to use the relation (25) between $f$ and $h$ to obtain the formula we wanted to prove:

$$
\frac{d h}{d t}(t)=\left[U_{r} \frac{\partial(\zeta+c \psi)}{\partial U_{r}}-(\zeta+c \psi)\right]_{\mathcal{L}(t)} .
$$

Quite remarkably, the $\zeta$-terms describing the twisting of the field lines by the boundary motions appear in that formula on an equal footing with the $\psi$-terms describing the dissipation by non-ideal effects. Note that the latter can also be written $\left(U_{r} \partial_{U_{r}} \mathcal{N}-\mathcal{N}\right)$, with $\mathcal{N}$ defined by equation (45). Of course, we recover that $d h / d t=0$ when $\zeta=0$ and $\mathbf{N}=0$, i.e., when the field lines are frozen in the plasma and their footpoints on $S$ do not move.

For comparison, we show a more general formula that holds true when the restrictive assumptions made at the beginning of this subsection are given up (this formula will be discussed in details elsewhere). If the reference vector potential $\mathbf{C}_{r}$ (not necessarily of the simple form used above) and the flux distribution on $S$ are allowed to depend on time, then

$$
\begin{aligned}
\frac{d h}{d t}(t)= & {\left[\left(\nabla_{s} \theta+\hat{\mathbf{n}} \times \nabla_{s}(\zeta+c \psi)\right) \cdot \frac{\mathbf{C}_{r s}}{B_{n}}-(\zeta+c \psi)\right]_{\mathcal{L}(t)} } \\
& +\int_{\mathcal{C}(t)}\left(\frac{\partial \mathbf{C}_{r s}}{\partial t}+\hat{\mathbf{n}} \times \nabla_{s} \theta\right) \cdot \mathrm{d} \boldsymbol{l},
\end{aligned}
$$


where $\mathcal{C}(t)$ is a curve of $S$ that connects the two footpoints of $\mathcal{L}(t)$ and we have used the general Helmholtz decomposition $B_{n} \mathbf{v}_{s}=\hat{\mathbf{n}} \times \nabla_{s} \zeta+\nabla_{s} \theta$ on $S$.

\section{Conclusion}

Let us summarize the results we have reported in this paper and indicate some of the extensions that are currently being worked out and will be presented in a forthcoming paper.

(i) We have established new formulae for the helicity $H$ of a line-tied magnetic field $\mathbf{B}$ relative to a reference field $\mathbf{B}_{r}$ and for its line helicity $h$ relative to a vector potential $\mathbf{C}_{r}$ of $\mathbf{B}_{r}$. These formulae relate these quantities to the magnetic mapping $\mathbf{M}$ of $\mathbf{B}$ and exhibit explicitly the topological invariance of $H$ and $h$. They are valid in the case where $\mathbf{B}$ has a simple topology and the part $S^{0}$ of $S$ where $B_{n}=0$ is a curve bounding both $S^{+}$and $S^{-}$. They can be generalized in two directions. Firstly, one can consider complex topology fields, for which $D$ can be decomposed into cells $D_{k}$ inside which the topology is simple and equation (13) still holds if we put an additive constant $c_{k}$ in its right-hand side. The main problem is then to relate the $c_{k}$ to $\mathbf{M}$. On the other hand, one can consider STFs for which $S^{0}$ is an annular domain (of positive area) separating $S^{+}$and $S^{-}$. The complication arising in that case stems from the fact that the topology of $\mathbf{B}$ is no longer characterized by the magnetic mapping alone. To fix the topology, one also needs giving a number $n_{w}$, which measures the winding of a magnetic line on $S^{0}$ (see Aly 2014 for a particular example of such a situation).

(ii) We have also given formulae for $h$ and $H$ in which intervene only the boundary values of a pair $(U, V)$ of Euler potentials of the admissible $\mathbf{B}$. Then these formulae make apparent, as those in terms of $\mathbf{M}$, the topological invariance of $h$ and $H$. A direct extension of these formulae to complex topology fields appear at first sight problematic as it is not possible to define global Euler potentials for such fields. But for the least one can extend them to the case of an STF for which $S^{0}$ is an annulus of positive area.

(iii) We have discussed the possibility that line helicity $h$ (along with the distribution of $B_{n}$ on $S$ ) characterizes the topology of the lines of an admissible $\mathbf{B}$. We have shown that this is the case if $h$ is relative to a reference vector potential of the form $\mathbf{C}_{r}=U_{r} \boldsymbol{\nabla} V_{r}$, with $\left(U_{r}, V_{r}\right)$ specific Euler potentials of an admissible reference field. A result of the same type was previously obtained by Yeates and Hornig (2014), who restricted their attention to tube-like STFs occupying a domain whose boundary $S$ is made of two horizontal planar domains $S^{+}$and $S^{-}$, and a vertical surface $S^{0}$, thus not threaded by the field. They found that two fields $\mathbf{B}$ and $\mathbf{B}^{\prime}$ that have $B_{n}=B_{n}^{\prime}$ on $S^{ \pm}, h=h^{\prime}$ for a specific choice of $\mathbf{C}_{r}$, and $n_{w}=n_{w}^{\prime}$, are topologically equivalent. Our argument can be extended to handle such a situation, actually without the geometrical restrictions above, and it is possible to show that 
two STFs with $B_{n}=B_{n}^{\prime}$ on $S^{ \pm}$and $h=h^{\prime}$ relatively to $\mathbf{C}_{r}=U_{r} \boldsymbol{\nabla}_{s} V_{r}$, have $\mathbf{M}=\mathbf{M}^{\prime}$ and $n_{w}=n_{w}^{\prime}$. Hence they are topologically equivalent.

(iv) We have considered the rate of change of the helicity of a line, followed in its motion, in the case where the plasma is moving on the boundary and/or non-ideal processes are acting in the domain $D$. We have derived a formula for $\mathrm{d} h / \mathrm{d} t$ valid when the flux distribution on the boundary is time-independent. More general situations can be handle along the same line, and we have now extended our calculations to the case where $B_{n}$ is no longer constant (an example of formula obtained in that case has been quoted at the end of subsection 6.2), and (as yet with some restrictions) to the case where the topology of the field is complex.

(v) Finally, we point out the existence of an analogy between the line helicity $h$ and the quantity $w=\int_{\mathcal{L}} B \mathrm{~d} l$ in the case where $\mathbf{B}$ is force-free in $D$ (i.e., $\boldsymbol{\nabla} \times \mathbf{B}=\alpha \mathbf{B}$ for some function $\alpha$ ). It turns out that $w$ can be related to $\mathbf{M}$ by a formula that is formally identical to equation (13) for $h$ (with $\mathbf{B}_{s}$ being substituted for $\mathbf{C}_{r s}$ ). This type of relation for $w$ allows (in combination with virial-type relations) to establish force-free energy formulae with a topological flavor (see Aly (2005, 2014) for examples implying a tube-like field). Topological energy formulae can be also obtained by using an Euler representation of B (Aly 1990). All these aspects are being currently developed.

\section{Acknowledgments}

I thank the two referees for their careful reading of my paper and for their helpful remarks.

\section{References}

Aly J-J 1990 Quasi-static evolution of a force-free magnetic field Comp. Phys. Comm. 59 13-20

Aly J-J 2005 A uniqueness result for a simple force-free equilibrium submitted to a topological constraint Astron. Astrophys. 429 15-18

Aly J-J 2014 Lower and upper bounds on the energy of braided magnetic fields J. Phys.: Conf. Series 544012003

Berger MA 1988 An energy formula for nonlinear force-free magnetic fields Astron. Astrophys 201 $355-361$

Berger MA 1986 Topological invariants of field lines rooted to planes Geophys. Astrophys. Fluid Dynamics 34 265-281

Berger MA and Field GB 1984 The topological properties of magnetic helicity J. Fluid Mech. 147 133-148

Démoulin P and Pariat E 2009 Modelling and observations of photospheric magnetic helicity Adv. Space Research 43 1013-1031

Finn JM and Antonsen TM 1985 Magnetic helicity: what it is and what it is good for? Comments Plasma Phys. Controlled Fusion 9 111-126

Moffatt KH 1969 The degree of knottedness of tangled vortex lines J. Fluid Mech. 35 117-129

Pevtsov AA, Berger MA, Nindos A, Norton AA and van Driel-Gesztelyi L 2014 Magnetic helicity, tilt, and twist Space Sciences Rev. 186 285-324 
Prior C and Yeates AR 2014 On the helicity of open magnetic fields Astrophys. J. 787100

Russell AJB, Yeates AR, Hornig G, and Wilmot-Smith AL 2015 Evolution of field line helicity during magnetic reconnection Phys. Plasmas 22032106

Schindler K 2006 Physics of Space Plasma Activity (Cambridge: Cambridge University Press)

Stern DP 1970 Euler potentials American J. Phys. 38 494-501

Titov VS, Hornig G and Démoulin P 2002 Theory of magnetic connectivity in the solar corona $J$. Geophys. Research 1071164

Woltjer L 1958 A theorem on force-free magnetic fields Proc. National Academy of Science 44 489-491

Yeates AR and Hornig G 2014 A complete invariant for braided magnetic fields J. Phys.: Conf. Series 544012002

Yeates AR and Hornig G 2016 The global distribution of magnetic helicity in the solar corona Astron. Astrophys. $\mathbf{5 9 4}$ A98

Zweibel EG and Li HS 1987 The formation of current sheets in the solar atmosphere Astrophys. J. 312 423-430 